\title{
A Novel Assessment of Delayed Neutron Detector Data in CANDU Reactors
}

\author{
Will Aylward \\ Department of Electronic \& Electrical Engineering \\ University of Strathclyde \\ Glasgow, Scotland, UK \\ Email: william.aylward@strath.ac.uk \\ Christopher Wallace \\ Department of Electronic \& Electrical Engineering \\ University of Strathclyde \\ Glasgow, Scotland, UK \\ Email: christopher.wallace@strath.ac.uk \\ Graeme West \\ Department of Electronic \& Electrical Engineering \\ University of Strathclyde \\ Glasgow, Scotland, UK \\ Email: graeme.west@strath.ac.uk \\ Curtis McEwan \\ Bruce Power \\ Tiverton, Ontario, Canada
}

A common opportunity for nuclear power plant operators is ensuring that routinely collected data is fully leveraged. Exploiting data analytics can enable improvements in anomaly detection and condition monitoring by identifying previously unseen data trends and correlations without major financial investment.

One such opportunity is in facilitating the detection of fuel defects by augmenting the delayed neutron monitoring system deployed in the majority of CANDU reactors. In this paper we demonstrate using archive data that the detection of fuel defects can be accelerated using this system in combination with the use of a deeper historical dataset and the introduction of a smoothing algorithm.

The current defect identification process relies on the analysis of data of high variance and is subject to the judgement of a domain expert, resulting in variable defect identification periods. The proposed approaches seek to mitigate this and alleviate the variable identification time. Initial results presented here show that for an initial batch of 30 defects, identification periods can be meaningfully reduced compared to the current process, with defects potentially visible an average of 11.4 days earlier.
By shortening this identification period, fuel containing defects can be scheduled for earlier removal, reducing the risk of statutory shutdown obligations, protecting personnel and promoting industry best practice.

Exploring a historical dataset identifies previously undocumented trends and we discuss the potential to produce correlations with other reactor parameters. The application of this knowledge can lead to opportunities in the use of machine learning algorithms and, ultimately, more accurate predictions.

\footnotetext{
Nomenclature

$2 \mathrm{xN}$ Double normalization

$\alpha$ Exponential smoothing factor

Br-87 Bromine-87

$C_{i, t} \quad$ Difference between cumulative sum \& total sum of $r_{i, t}$ D Defect detection point

DN Delayed neutron

$\Delta \operatorname{var}\left(X_{i}\right) \quad$ Change in $2 \mathrm{xN}$ variance before/during defect period

$E_{\text {est }, t} \quad$ Estimation error at time $t$
} 
$E_{\text {meas.t }}$ Inherent measurement error

ES Exponentially smoothed

$E S T_{t} \quad$ Filtered estimate at current time $t$

GFP Gaseous fission product

I-137 Iodine-137

$K G_{t} \quad$ Kalman gain at current time $t$

$\mathrm{MW}_{e l} \quad$ Electrical power output

$r_{i, t}$ Array of differences between channel $2 \mathrm{xN}$ values \& channel group mean values

STD Standard deviation

$\operatorname{var}\left(X_{i, \text { defect }}\right) \quad 2 \mathrm{xN}$ variance during defect period

$\operatorname{var}\left(X_{i, p r e}\right) \quad 2 \mathrm{xN}$ variance before defect period

$X_{i, p o s t} 2 \mathrm{xN}$ values recorded during defect period

$X_{i, p r e} 2 \mathrm{xN}$ values recorded prior to defect period

$X_{i, t} \quad 2 \mathrm{xN}$ values

$x_{t} \quad$ Measured $2 \mathrm{xN}$ count value at time $t$

\section{Introduction}

Data is constantly gathered at nuclear power plants, and is generally used to help engineers and operators make key decisions regarding specific plant operations. While operators will have a deep technical knowledge of the systems they are managing, there is often limited re-use or statistical analysis of data once it has served its initial purpose. As a result of advances in machine learning which have not yet been fully leveraged in many operational areas, there exists an opportunity to improve the decision making process so that faults may be detected earlier, more accurately and with fewer dedicated personnel hours. These advances can also enable larger historical datasets to be leveraged, and combining this with novel analytics can lead to a deeper understanding of the core behaviour at little extra cost to plant operators.

Here we present the initial assessment and analysis of data from one such area: that of delayed neutron monitoring for fuel defect detection in CANDU nuclear plants.

This paper is divided into the following sections: in Section 2, CANDU reactors and the motivation for fuel monitoring are described and one current analysis process is subsequently introduced. We outline our general approach to the analysis in Section 3 and provide more detail on this in Sections 4, 6 and 8. Results and discussion follow their respective descriptions in Sections 5 and 7 and we conclude by suggesting further work of interest.

\section{CANDU Reactors}

At the time of writing, 31 commercial CANDU reactors of various sizes operate globally. 18 of these, the 480channel CANDU-9 variant, are located in Ontario, Canada with an output of approximately $850 \mathrm{MW}_{e l}$ per unit. Of the other units, the most common is the 380-channel, approximately $650 \mathrm{MW}_{e l}$ CANDU-6.

Fuel in CANDU-6 and CANDU-9 reactors is arranged in bundles, each containing 37 fuel elements, with 12 or 13 bundles in each of the fuel channels. All are orientated horizontally, and individually pressurized. One advantage of the design is that every fuel channel can be individually refuelled without taking the reactor offline [1].

\subsection{Fuel Monitoring Motivation}

Components within CANDU reactor cores are subject to high levels of stress, primarily from the intensity of heat created in the fission process, however the heat fluctuation and high flow speed of the heavy water coolant are also major contributors.

Fuel elements comprise around 30 uranium oxide pellets within a thin zircalloy sheath. Ongoing exposure to fast neutrons causes embrittlement and weakness of this sheath. Upon refuelling, the coolant is allowed to carry the new fuel bundle into the reactor, resulting in unavoidable collisions between new and old fuel bundles, leading to another source of materials stress [1].

As a result of the stresses the fuel is exposed to, defects can occasionally occur. The frequency of events such as these in CANDU reactors are some of the world's lowest amongst water cooled reactors [2], but for personnel protection and operational requirements it remains important that they are detected and removed quickly.

When a fuel defect occurs, fission byproducts are able to escape into the coolant. Some of these byproducts have long half-lives and can cause long term contamination of the primary coolant loop, which has specific operating limits for radiation levels [3]. Fuel bundles with multiple defects can be more difficult to remove from the reactor if excessive deformation is allowed to progress [1], which further incentivizes the identification and location of defects at an early stage.

\subsection{Fuel Monitoring Systems}

The detection and location of fuel defects in the majority of CANDU reactors is achieved using two systems [4]. The first monitors the primary coolant for the presence of gaseous fission products (GFP) and specific radionuclides and is used to indicate the presence of a fuel defect somewhere within the core. The second system is deployed periodically and uses the emission of delayed neutrons (DNs) to identify the channel containing defect fuel. The data generated by this second system is the focus of the work presented here, but the information derived from the GFP system is useful when interpreting the DN data and will be referenced later.

In this paper we focus on the data available from the eight CANDU-9 units operated by Bruce Power. Here, the 480 channels are monitored via two measurement halls; one at each end of the reactor and each containing 240 sampling points. Measurement points for each of the 240 channels are arranged in an $8 \times 30$ array in each measurement hall. A rig comprising 8 neutron detectors moves horizontally across this array, stopping for a short period at each sample point to simultaneously collect 8 neutron count values [5]. The collection of DN activity data for all 240 channels in a single session in a single hall is now referred to as a 'scan'.

If a defect occurs, fission byproducts are released into the primary coolant loop. DNs are released and detectable 


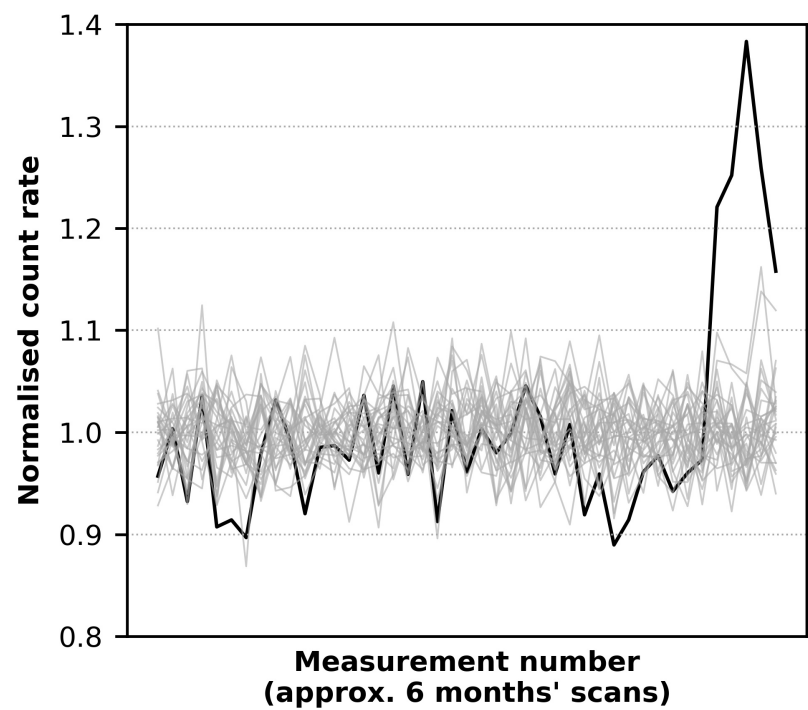

Fig. 1. A representative DN plot, with defect channel highlighted

in monitoring halls outside the reactor, predominantly via I137 with half-life of 22 seconds and $\mathrm{Br}-87$ with half-life of 56 seconds [3]. Inside the reactor, DNs from escaped fission products make up only approximately $3 \%$ of total activity, however a short time after leaving the core they account for $75 \%$ of DN activity due to the longer half-lives of these specific fission byproducts. As a result, it is possible to detect which channel contains a fuel defect by observing elevated neutron activity levels.

After the coolant has passed through the measurement hall, it is returned to the reactor and recirculates. To minimize noise from recirculated, longer lived fission products, the system is designed such that the coolant transport time from fuel channel to DN detector maximizes the detection of DNs from the target isotopes I-137 and Br-87 [3].

Plant conditions also play an important role in the release of DN precursors [4] and can delay the presence of DNs at the monitoring location, and hence apparent activity spike, for several days or weeks until sufficient levels of fission byproduct are leached into the coolant.

\subsection{Current Analysis}

Bruce Power employs a Double Normalization $(2 \mathrm{xN})$ technique. For each detector, count values are normalized with respect to the average count rate for the 30 channels in question. This removes most effects of any settings changes and changes in background neutron count between scans and goes some way towards removing any inter-monitor bias, assuming all monitors are connected to similarly active channels, as the design intends. Secondly, data is filtered and normalized channelwise, so if one channel consistently produces a higher reading than others, this bias will be removed [6].

This technique is applied to every group of channels for all 8 detectors, and by plotting these values any anomalous channels can be identified as their double normalized counts will usually appear to trend higher than their neighbours.
Figure 1 shows an example of data from a group of channels, with the highlighted data series derived from a channel containing defect fuel. In this example, the defect appears quickly and is clearly identified but this is not always the case. The defect channel identification process is challenging as the data has low resolution and high variance and operating experience using the existing $2 \mathrm{xN}$ technique has demonstrated that the time taken for the channel containing the fuel defect to become distinguishable from its neighbouring channels varies considerably. Greater clarity and repeatability in this area would be beneficial.

Fuel defect detection in CANDU reactors has been the focus of some previous work. [7] summarized the current approach based on calculation and thresholding of a DN discrimination ratio, comparing count rates to background. The employment of this technique is described at CANDU sites in New Brunswick [8], South Korea [9], Romania [10], Pakistan [11] and India [12]. The $2 \mathrm{xN}$ technique [6] is a direct development of this method. Other work [13] has focussed on improved online decision support for the GFP system using a physical understanding of the behaviour of specific radionuclides to more quickly identify anomalous reactor parameters and other studies have refined this progress to account for the effect of equipment fouling on the sensitivity of the DN detectors [14]. To the authors' knowledge, no recent work has been undertaken to specifically improve the DN time series analysis.

\section{General Approach}

The focus of this work is the assessment of online DN monitoring data, with the aim of reducing the time taken between initial detection of an in-core defect to scheduling its removal.

Figure 2 outlines the development of this work, which is divided into two stages. In stage 1, an initial review of a small sample of defects was undertaken and the potential for improvement in the existing defect localisation process was explored. In stage 2, a more extensive database was reviewed for bulk historical analysis, with the aim of visualising, identifying and incorporating longer term trends. Any observable trends can then be identified and validated using station operational knowledge.

In parallel with the historical analysis, a smoothing process is used to mitigate the effects of noise in the dataset. Knowledge gained in the historical analysis is expected to be incorporated into the smoothing process in future work and is therefore represented as a dotted connector.

The limited initial release of DN data referred to in Fig. 2 covered several units at the Bruce Power site, for time periods around specific fuel defects of approximately one year. The full data release covered all 8 units, spanning various time periods up to 30 years. In total, each reactor has between 500-800 fully-labelled scan files, containing one data point for each channel. 


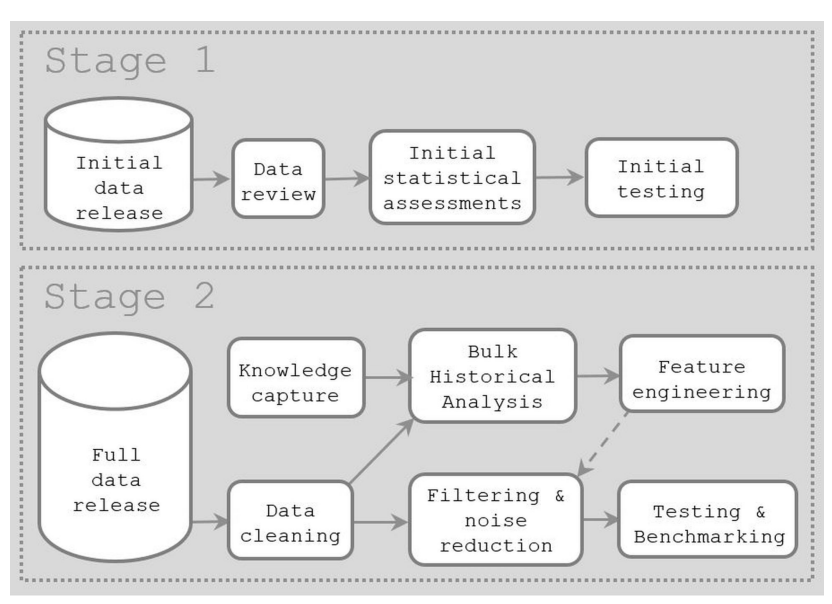

Fig. 2. General approach for the analysis of DN data

\section{Initial approach: Statistical Assessments}

Using a small representative set of data with labelled defects, preliminary assessments explored the application of alternative anomaly detection methods. The double normalized dataset $X_{i, t}=x_{1, t}, x_{2, t}, \ldots, x_{30, t}$ was split into $X_{i, p r e}$, the data recorded prior to defect detection and $X_{i, d e f e c t}$, the data recorded after detection but before channel identification for the 30-channel monitor group containing the defect channel.

\subsection{Change in Mean}

One approach was to calculate the change in mean of the count rate for each channel, reasoning that on average, the count rate should increase for a channel containing a defect and so the comparison of the mean of two similar subsequences before and after the GFP detection date should be robust to the presence of noise and may help to highlight this discrepancy. Any trends in $X_{i, p r e}$ can be difficult to see by human inspection and so this metric may emerge before the raw $2 \mathrm{xN}$ values reached any threshold applied. For channel $i=1$ to 30 ,

$$
\Delta \overline{X_{i}}=\overline{X_{i, d e f e c t}}-\overline{X_{i, p r e}}
$$

At each time point in the defect period, the $2 \mathrm{xN}$ values were recalculated and per-channel mean changes calculated. Any negative deviations are ignored on the basis that only an increased count is expected for a channel containing a defect.

\subsection{Change in Variance}

Another approach quantified the variance change of each channel from the point at which the GFP system identified an in-core fuel defect. The deviation in the distribution of data points either side of the GFP detection date for a series could indicate a defect for that channel. For channel $i=1$ to 30 ,

$$
\Delta \operatorname{var}\left(X_{i}\right)=\operatorname{var}\left(X_{i, \text { defect }}\right)-\operatorname{var}\left(X_{i, p r e}\right)
$$

\subsection{Cumulative Residuals}

On the basis that small differences from the mean behaviour may not be visible due to high levels of noise, it is proposed that the cumulative effect of these differences may be more obvious. This is a common strategy in anomaly detection methods, employed in cumulative summation charts first by [15] which continue to be used to this day.

With each new scan, the final method shown calculates an array of differences between the counts and the average count for all channels in the window since the GFP detection.

$$
r_{i, t}=X_{i, t}-\overline{X_{d e f e c t}}
$$

The cumulative sum and total sum of these differences is then calculated, with the difference between these, $C_{i, t}$, given as

$$
C_{i, t}=\sum_{0}^{T}\left(r_{i}\right)-r_{i, t}
$$

In this way the final value is always 0 (because the final cumulative sum = total sum) and any anomalously high counts are expected to trend above the others when plotted graphically. A window starting at point of GFP detection can be analysed, with the exact starting point adjusted to optimize detection capability.

The following section provides some initial results from application of the analysis techniques outlined above.

\section{Results: Statistical Assessments}

Figures 3 and 4 show the results of applying the change in mean and change in variance calculations. The upper panels display the $2 \mathrm{xN}$ data, with the data from the defect channel highlighted as a black dashed line. The date at which an in-core defect was detected by the GFP system is shown as the vertical dash-dotted line, with the date at which the defect channel was identified with enough confidence to justify defuelling the channel by analysis of the $2 \mathrm{xN}$ data shown as the vertical dotted line.

The middle panels show the changes in mean between the pre-defect data and the post-defect data for each channel at that point in time. For these plots, the vertical dotted lines from the upper panels have been retained for reference, with only the defect period shown. Again data from the defect channel is highlighted as a dashed line. In both figures, the defect channels are identifiable by this parameter multiple scans earlier than by the $2 \mathrm{xN}$ data alone. For defect example 1, the defect is differentiable from the beginning of March, which represents a substantial improvement of almost 8 weeks over the current method. For defect example 2 , the defect is visible from the beginning of August, representing an improvement of around six weeks.

The lower panels show the changes in variance for the individual double normalized count data. In these cases, the 
2xN data: defect example 1

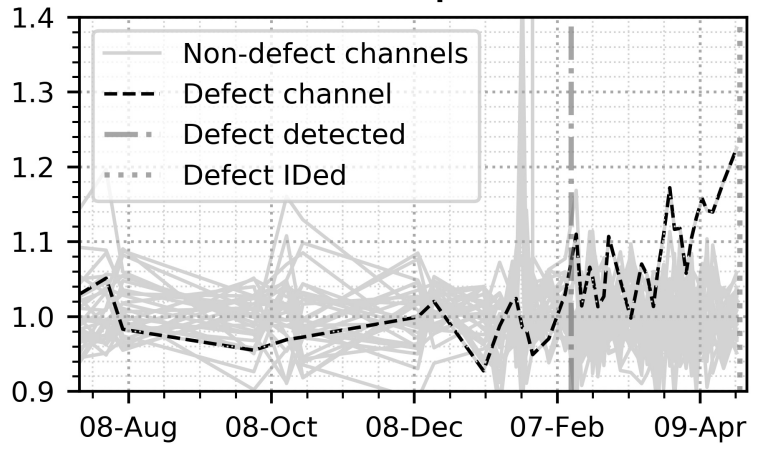

Change in mean

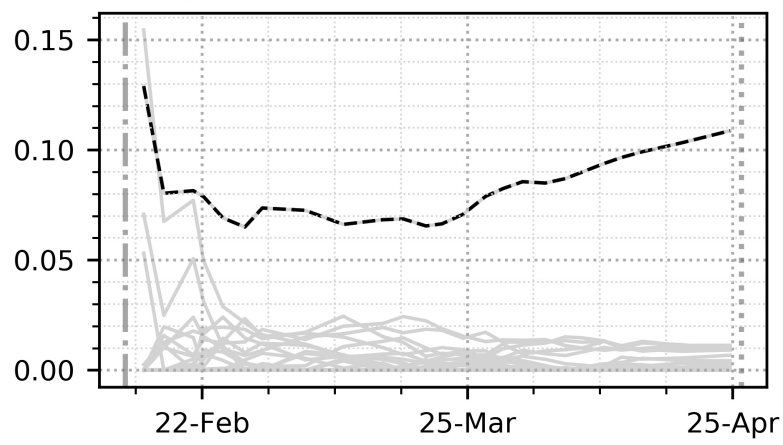

Change in variance

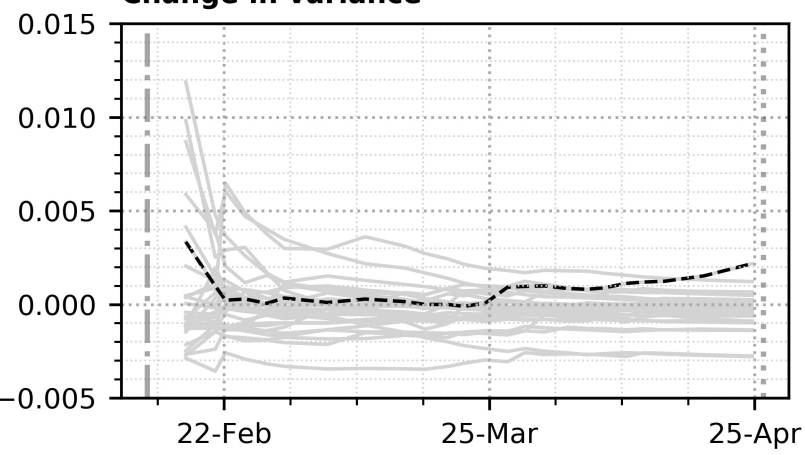

Fig. 3. Calculated metrics for defect example 1

variance changes are not an obvious early indication of a channel containing a defect, but initial observations suggest that defect channels tend to trend amongst the highest of their monitor group, especially as the channel identification date approaches. On its own, the change in variance metric is not immediately useful, but it may prove a useful predictor in combination with other features.

Figure 5 displays the results of the cumulative residual assessment for defect example 1, the same defect analysed in Fig. 3. Four panels are presented showing examples results of the analysis as they appear at various points in time. It should be noted that the manner in which the calculations are carried out does not allow a single plot (showing all time points) to be produced as in the preceding figures; instead figures are generated each time a scan is carried out. The defect identification date of April 26th is again shown by the vertical dotted line to the right, and snapshots are presented of the cumulative deviations 5,10,15 and 20 scans prior to this date, referred to on the plots as D. All available data is
2xN data: defect example 2

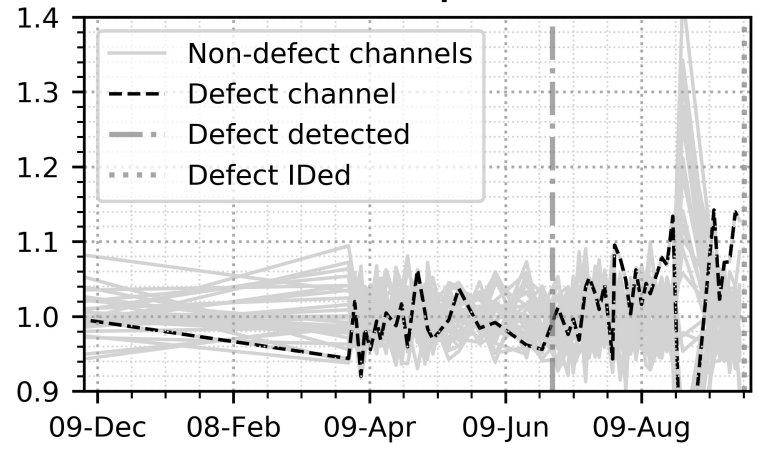

\section{Change in mean}
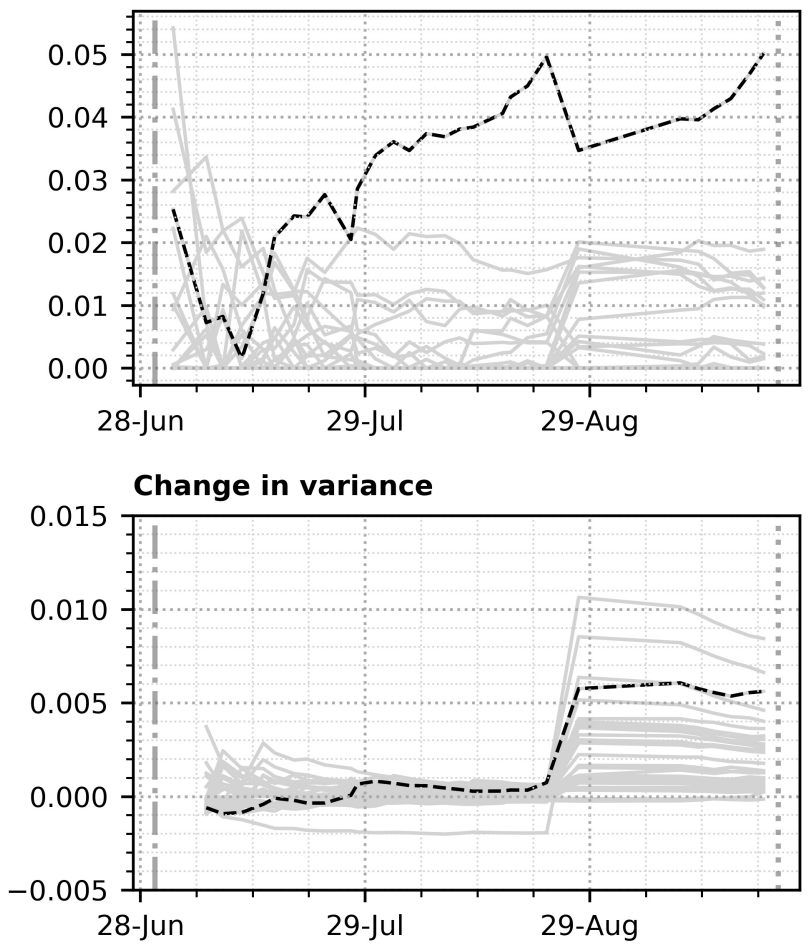

Fig. 4. Calculated metrics for defect example 2

plotted for each snapshot, so Fig. 3a would have been generated on April 7th. For this defect example the defect channel is again distinguishable from the non-defect channels by the greater magnitude of deviations at an early stage.

These early visualisations provided evidence that alternative approaches to the current analysis process were possible. Three methods have been presented here which show some promise for identifying anomalous channels multiple scans earlier than with existing methods. It should however be noted that the trends identified are not always consistent for all defect examples available and appear somewhat sensitive to the selection of pre-defect data period.

\section{Noise reduction techniques}

Two related signal processing techniques for the removal of noise are now discussed.

The Kalman filter is a well established recursive statistical method used to mitigate the effects of noise in data se- 

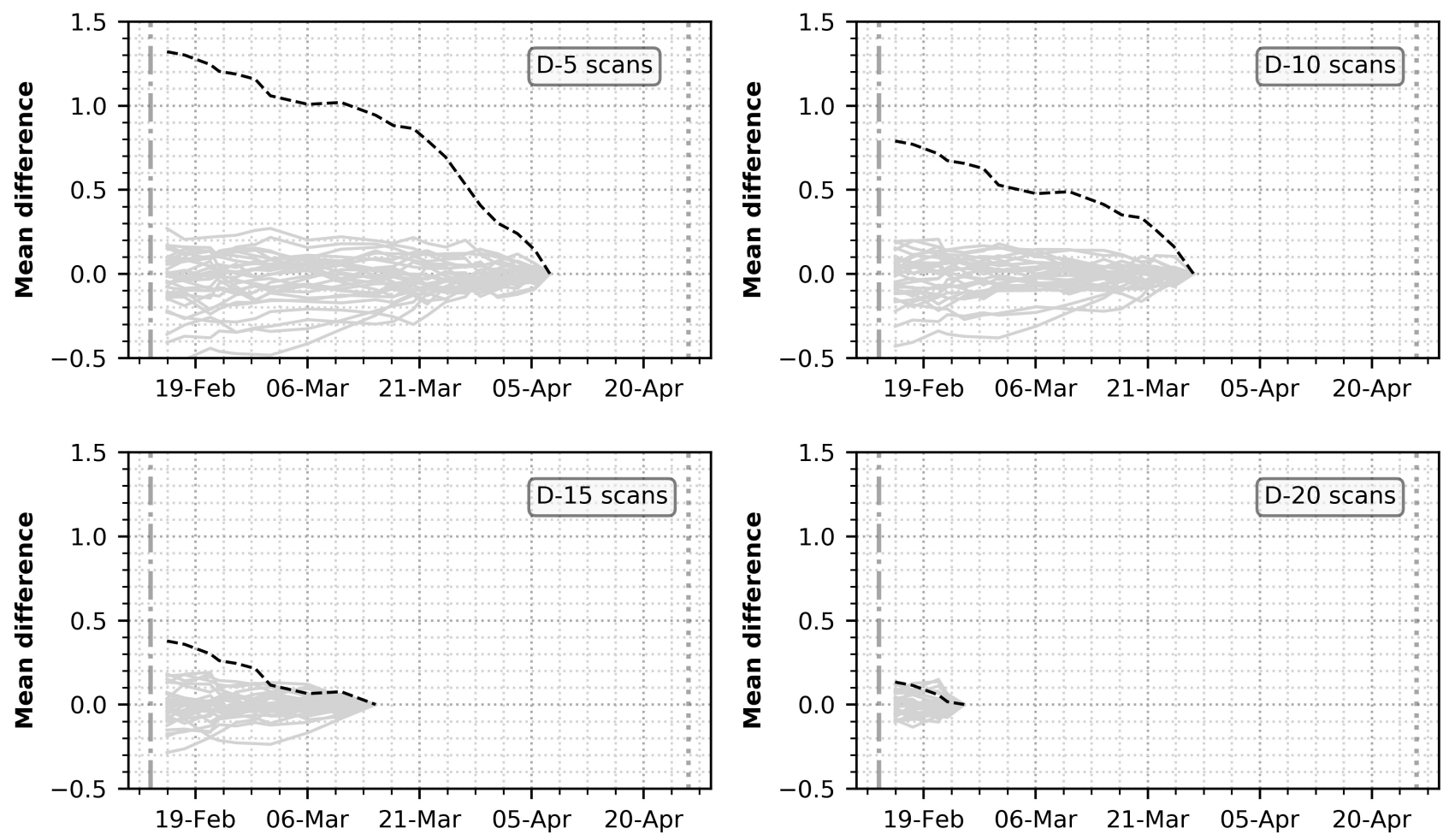

Fig. 5. Cumulative deviations from mean $5,10,15 \& 20$ scans from original defect identification point: defect example 1

ries, so investigation of its application was of interest. The univariate filter can be applied on a per-channel basis, as follows:

$$
K G_{t}=\frac{E_{e s t, t}}{E_{\text {est }, t}+E_{\text {meas }}}
$$

$$
E S T_{t}=E S T_{t-1}+K G_{t}\left(x_{t}-E S T_{t-1}\right)
$$

$$
E_{e s t, t}=\left(1-K G_{t}\right) E_{e s t, t-1}
$$

Where $K G_{t}$ is the Kalman gain at time $t, E_{\text {est }, t}$ is the estimation error at time $t, E_{\text {meas }}$ is the inherent measurement error (learned from pre-defect data subset), $E S T_{t}$ is the filtered estimate at time $t$ and $x_{t}$ is the measured count value at time $t$.

The inherent measurement error is not directly known; a common challenge when applying Kalman filter variants [16]. To estimate that error, we use a multiple of the interquartile range of the double normalized values recorded prior to the defect being detected.

This method shows some promise but as is typical with the Kalman Filter algorithm, the gain parameter reduces as more data is collected. This has the effect of giving lower weight to any newly recorded data point. As a result, the predictions become over-smoothed and any emergent defect takes time to appear by human inspection. This is a disadvantage where swift identification of an anomalous trend is the key driver, so work to address this limitation is ongoing.

Exponential smoothing (ES) is a processing technique in effect similar to the univariate Kalman filter with gain parameter held constant. It is defined as follows:

$$
E_{e s t, t}=\alpha \times x_{t}+(1-\alpha) E_{e s t, t-1}
$$

Where $\alpha$ is the smoothing factor and controls the weighting given to the latest measurement compared to the previous estimate, as with the Kalman gain described above. Typically this parameter is chosen in the range 0.1-0.3 [17]; here we use a value of 0.15 which was found to be a good compromise between sensitivity and noise reduction.

\section{Results: Noise Reduction}

A period of approximately three months is currently used to generate the $2 \mathrm{xN}$ data. Here the effect of extending the historical dataset used to generate the double normalized data, and smoothing the resultant values, is demonstrated. In Fig. 6, the upper panel shows data from the defect period only. Again, the GFP alarm date is shown as the vertical dash-dotted line while the defect identification date is shown as the vertical dotted line, both in grey. The $2 \mathrm{xN}$ data for the defect channel is shown as a dashed black line, with the $2 \mathrm{xN}$ 
data for the non-defect channels in the background in light grey. The exponentially smoothed values for the non-defect channel are shown on top of the $2 \mathrm{xN}$ values, in dark grey; the same data for the defect channel is highlighted as a solid black line with circular markers.

The lower panel shows a representation of the available data for the monitoring hall for that particular unit. The average count rate for all 240 channels for every monitoring session in each hall are displayed separately (NB monitoring sessions occur at different times in the East and West halls). The standard deviation for each monitoring session for the hall from which the defect channel is monitored is also displayed. Both mean and standard deviation were displayed in this way in order to identify any monitoring sessions which may have generated any abnormal values and which may need to be discarded. At this point, no filtering on this basis has been applied. The light grey shaded region identifies the time period from which the $2 \mathrm{xN}$ values were derived.

Now, all available historical data is incorporated: the results are shown in Fig. 7.

In this case, the defect channel appears above the other channels from the same monitor group and is clearly distinguishable as an outlier by inspection of the $2 \mathrm{xN}$ data at a very early stage: in this case from around the end of November. Again, this demonstrates an improvement of approximately eight weeks over the existing analysis process. The exponentially smoothed values are also displayed on these figures: for this example, this filtered data is not strictly necessary to identify the defect but does help to confirm the hypothesis strongly suggested by the $2 \mathrm{xN}$ data. In some cases, exponential smoothing in combination with history extension improves performance beyond the current approach using the $2 \mathrm{xN}$ technique. Figure 8 shows one such example which benefits from this smoothing process.

For this third defect example, the $2 \mathrm{xN}$ data generated by the original analysis window gave no indication of the location of the defect. Figure 8 shows the result of extending the window to cover all available data, with that data again used to generate exponentially smoothed predictions. In isolation, the double normalized values do not allow for clear visual distinction of the defect-containing channel as the signal is moving within the bounds of the variation of its neighbours. However, by inspecting the exponentially smoothed data series, the defect channel emerges from this noise around the beginning of January. While this technique may not provide enough evidence to comprehensively prove the existence of a defect in that channel, it should enable the creation of a significantly smaller set of candidate channels for review.

The defects examples shown here tend to illustrate some of the better performing channels using this method, so we quantify the results of the available set of data in Table 1. This table displays the number of channels in which various scan reductions were observed compared to manual inspection by a human expert. With a previous average defect location time of 41 scans for this set of defect examples, using this method it was found that defects are potentially visually identifiable on average 4.9 scans earlier than the exist-
Table 1. Summary of improvements enabled by exponential smoothing approach

\begin{tabular}{cl}
\hline Scans saved & \# examples \\
\hline 0 & 6 \\
$1-2$ & 7 \\
$3-4$ & 6 \\
$5-6$ & 5 \\
$7-8$ & 2 \\
$9-10$ & 2 \\
$10+$ & 2 \\
\hline
\end{tabular}

ing $2 \mathrm{xN}$ inspection process. For this set of defect examples this equates to 11.4 days, which represents a meaningful improvement.

\section{Data Exploration: Historical Visualisation}

As previously discussed, on completion of an initial review of a smaller dataset a more detailed historical analysis could also be made. Here we seek to identify longer-term trends which can enable the incorporation of more sophisticated analytics tools. Several years of data was parsed and extracted from the regular monitoring of 8 units. Feature information in a suitable archival form is currently limited but other trends of interest can nonetheless be identified.

As a first step, the mean, median and standard deviation of the raw data was plotted as a time series to visualize any long term trends. This is shown for one unit in Fig. 9. Each point relates to the average, median or standard deviation of a complete scan for a single monitoring hall. In general, there appears to be a tendency for average count rates to gradually reduce over time, with step changes following extended outages. Discussion with engineers indicates that the long-term downward trend may be due to the reduction of monitor sensitivity when exposed to neutron flux, and the step change potentially due to recalibration, cleaning or recovery of monitors. Similar trends are visible for most other units and investigations are ongoing.

Further insight can be gained by plotting this data as a heatmap for each measurement hall. An example is shown in Fig. 10, where every vertical slice corresponds to a single scan and every horizontal slice corresponds to a single channel of the 240. In order to perform comparisons between different detectors, it is necessary to first normalize the count data. A standard scaling process was selected to produce a distribution with zero mean and unit standard deviation across the date axis for each monitor group, which allows identification of inherent activity differences between channels. This is similar to the double normalization technique but in this case, anomalous values are not automati- 


\section{2xN \& Exponentially smoothed data - defect example 3}
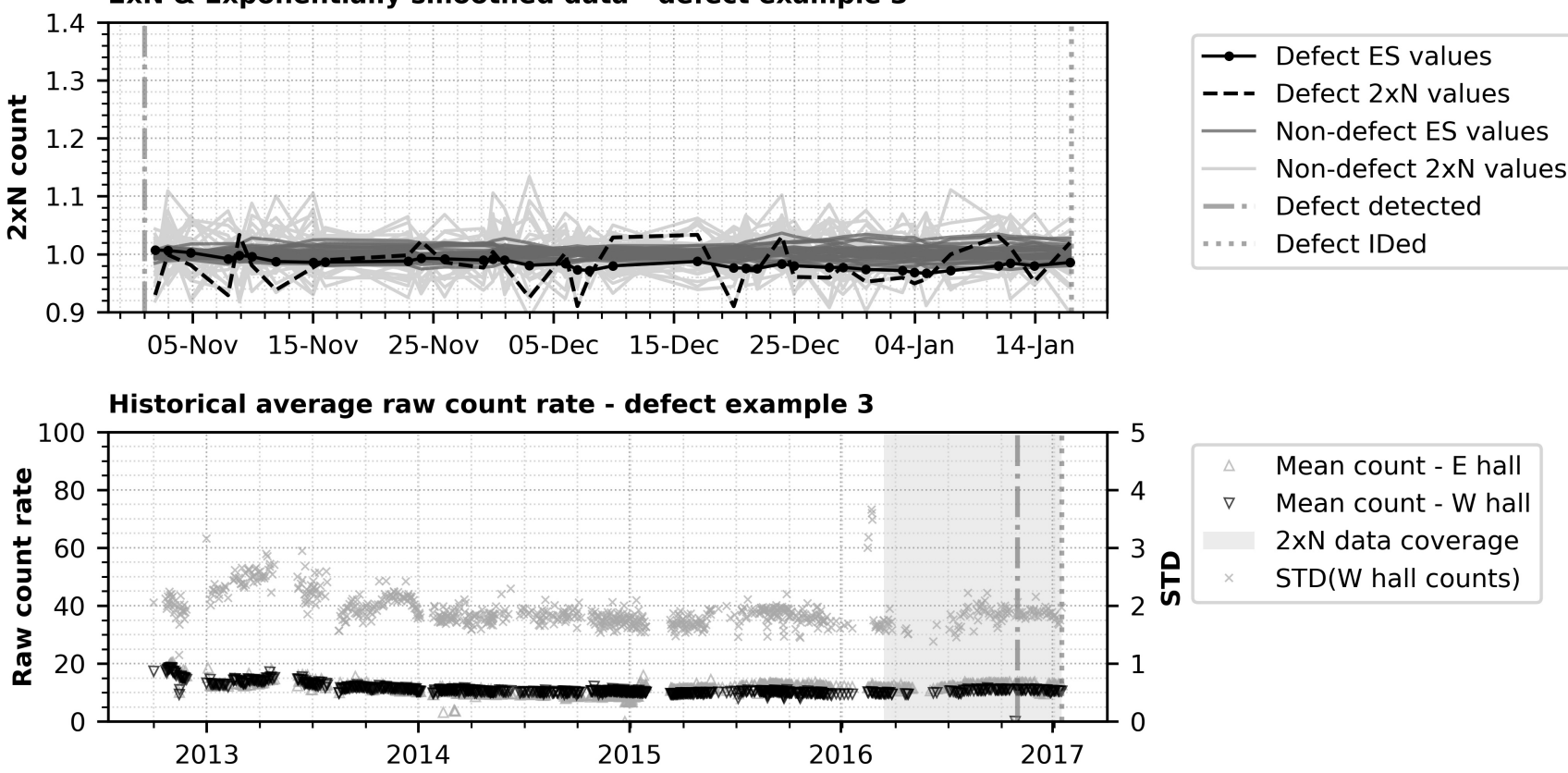

Fig. 6. 2xN \& ES data for defect example 3, original history
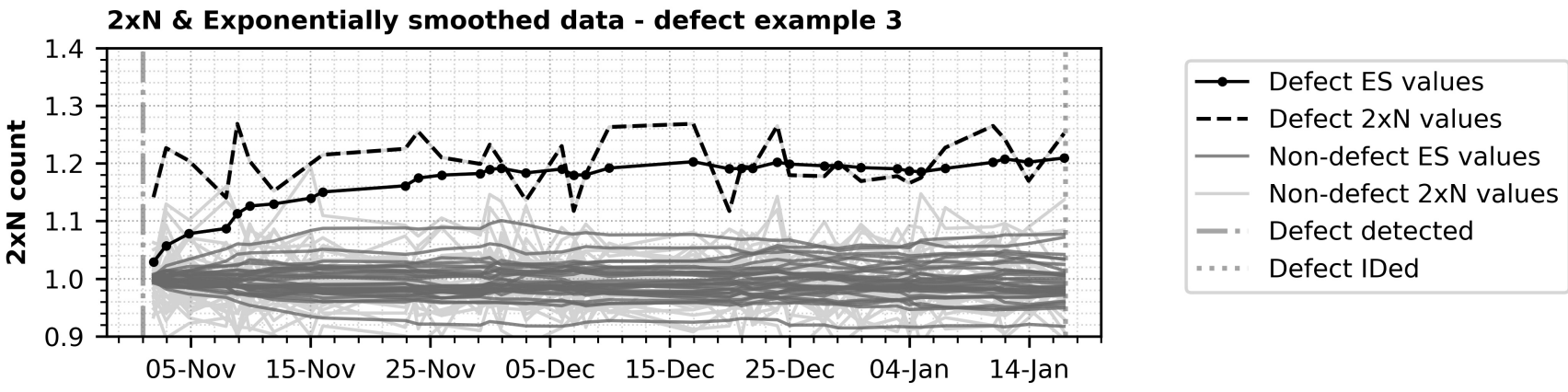

Historical average raw count rate - defect example 3

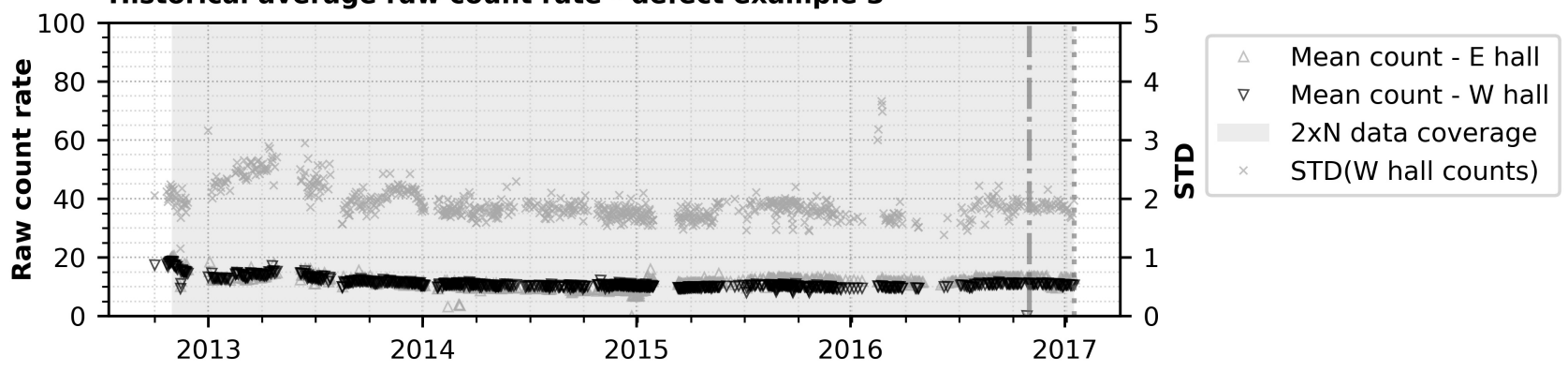

Fig. 7. $2 x N \&$ ES data for defect example 3, extended history

cally rejected and the values generated can be directly plotted to illustrate this spread. The arrangement of the data is such that on the $y$-axis, channels are grouped by detector. Within each detector band there is a graduated distribution in the normalized count from low to high. It is suspected that this pattern is the result of a couple of factors: one is referred to as the "north-south" effect, whereby the higher level of gamma shine at the end of the monitoring array closer to the reactor core leads to an increased signal at these locations. Secondly, when the system was designed there was an attempt made to ensure even distribution of low and high power channels across each detector. As with most reactor designs, central channels tend to have a higher associated power (and associated DN activity) than those on the periphery. It is likely a combination of these effects which leads to 8 horizontally repeating patterns.

Visualising the data in this manner for this particular unit has highlighted a clear, distinctly different response in one of the detectors: a number of anomalies are visible in the 6 th horizontal band which indicates that this monitor has oc- 


\section{2xN \& Exponentially smoothed data - defect example 4}
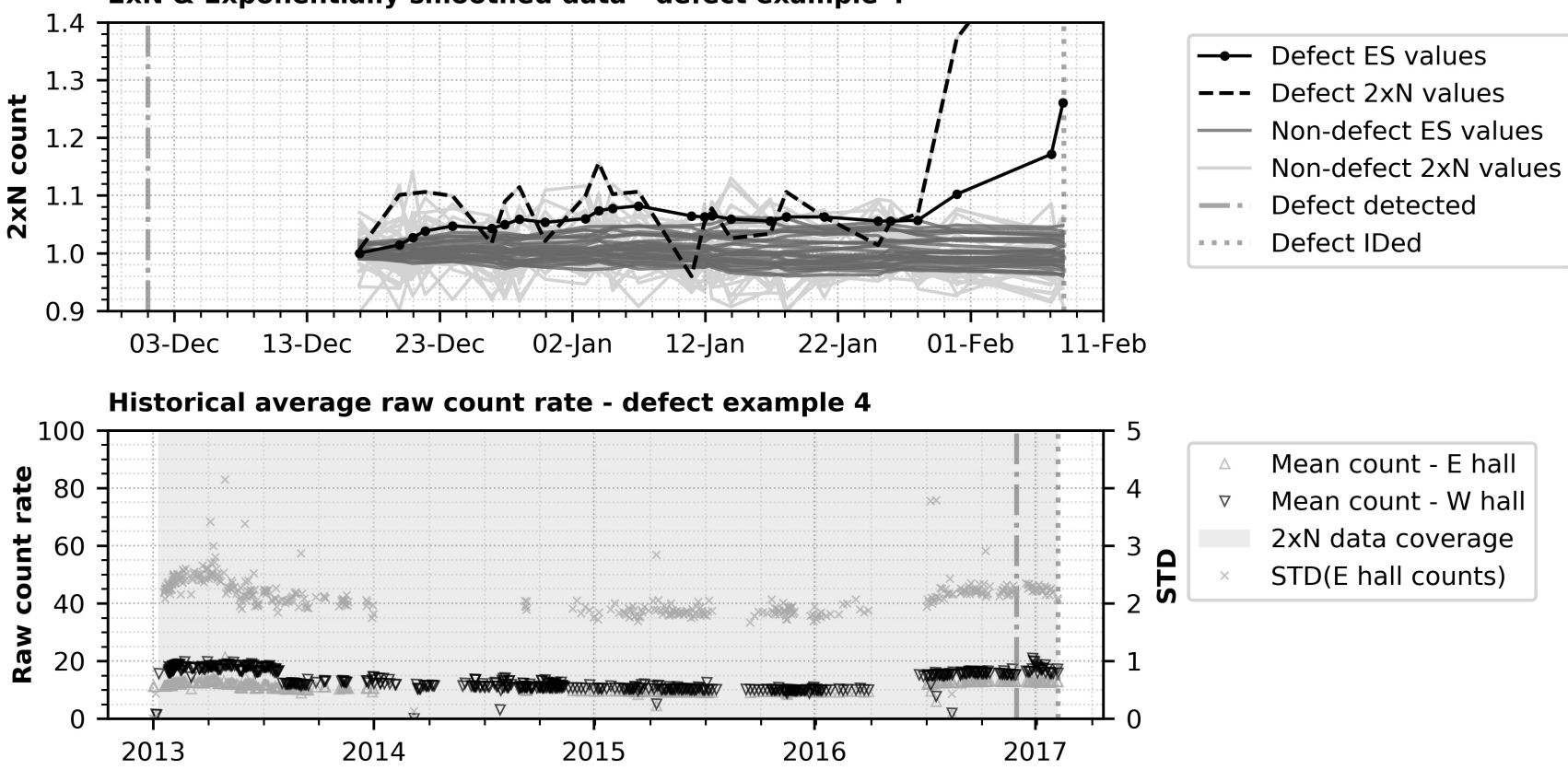

Fig. 8. 2xN \& ES data for defect example 4, extended history

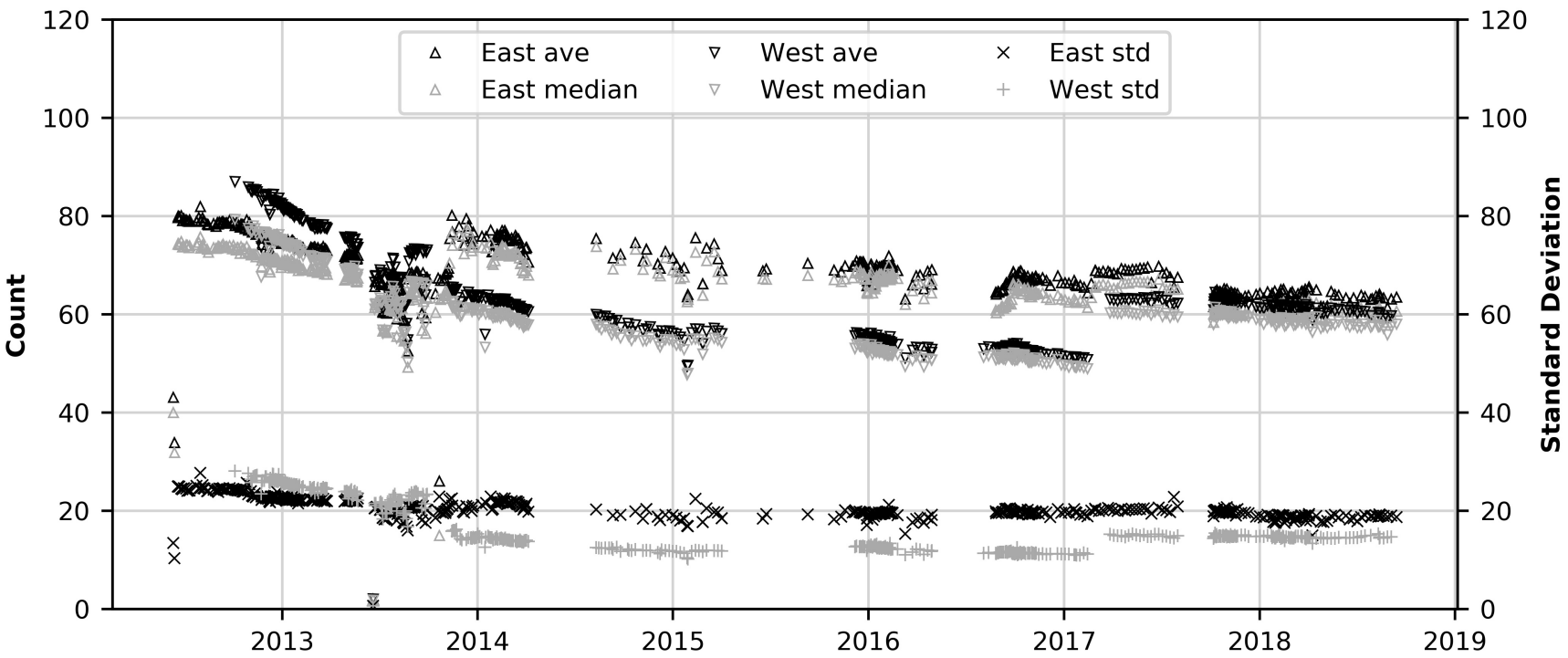

Fig. 9. Mean, median \& standard deviation of raw counts for a single unit

casionally been causing spurious readings for several years. This is not a critical issue as errors are almost always obvious and can be quickly discarded but further investigation is ongoing with station engineers to determine the root cause of this behaviour. It is worth noting that both Fig. 9 and 10 show approximately six years of inspection data on a single graph in a way which is not normally displayed and this has identified some previously unseen insightful trends.

An important aspect of any analysis techniques developed is to ensure that there is an understanding of the underlying physical processes, which involved close discussion with engineers at Bruce Power. In this way, insight has been gained regarding the physical layout of the pipework con- necting the reactor to measurement halls, as well as the difference in appearance of real defects compared with electronic errors. It is suggested that the long term general behaviour of the monitors may prove to be useful in identifying anomalies in the data. The incorporation of these effects into any future model will be instrumental in the feature engineering step identified in Fig. 2.

\section{Conclusions \& Future Work}

This paper has demonstrated the additional value that can be gained from the application of modern data analytic techniques to leverage existing data sources and provide additional insights into core behaviour, in this case using his- 


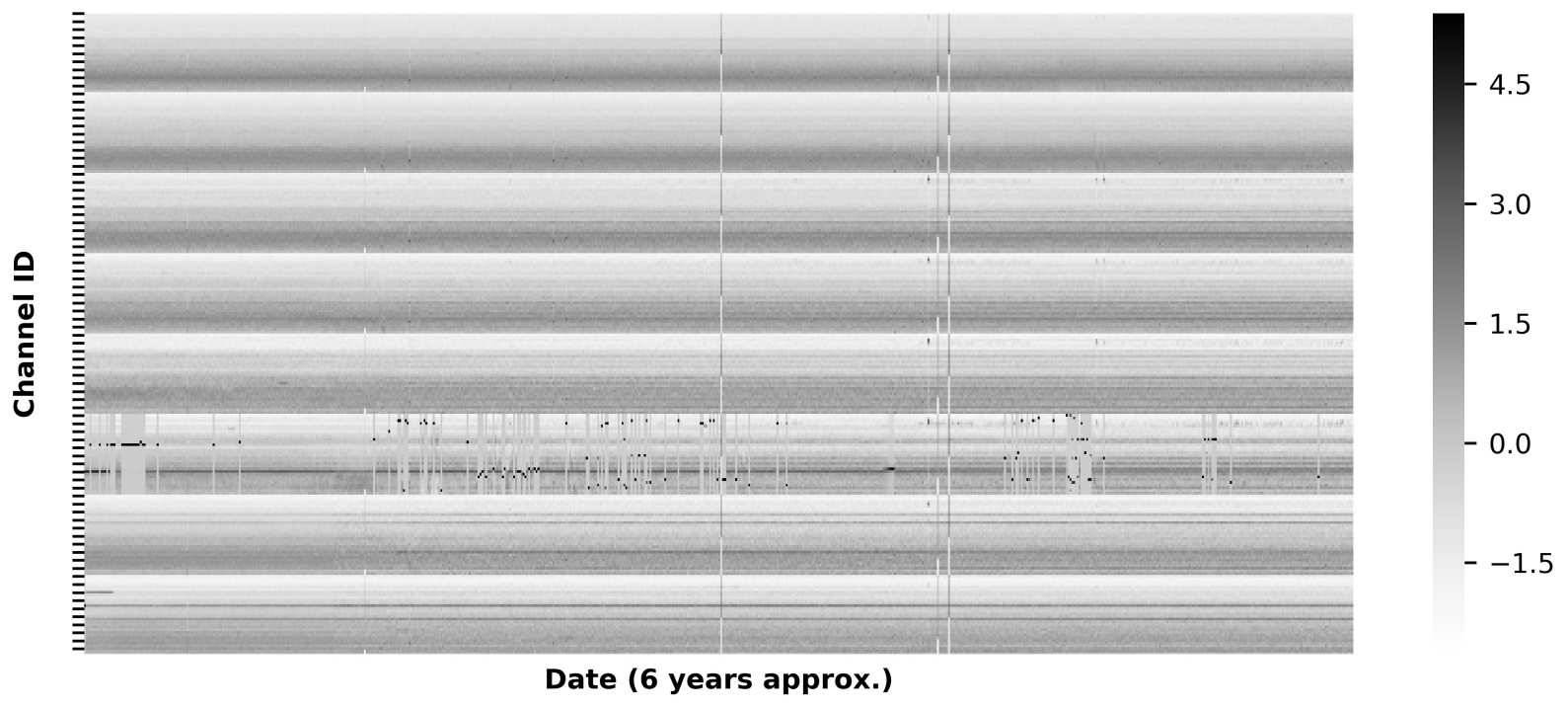

Fig. 10. Heatmap of normalized counts, scaled by monitor, shaded by standard deviation differences from mean

toric delayed neutron data.

An initial review of a number of approaches toward improved DN analysis have been presented. This work has proved promising in this regard, with some statistical analyses potentially able to identify channels containing fuel defects several weeks earlier than the current system. Further detailed analysis against other historical instances of defect fuel across different reactors may build a case for moving towards using one, or a combination, of these methods in conjunction with the double normalization technique and exponential smoothing.

Analysis of a larger dataset has enabled longer term trends in the data to be identified, and the use of exponential data smoothing techniques has shown promising results, with defects potentially identifiable an average of 11.4 days earlier than by using the current double normalization method. Some promise is also shown by the univariate Kalman filtering approach, although initial results have shown that the sensitivity is compromised at the expense of over-smoothing.

Good value has been demonstrated by visualising the historical dataset in the form of a heatmap and it is anticipated that other similar historical data sources, many of which exist in the nuclear domain, will likely benefit from this approach.

Further work could focus on the development of an algorithmic approach to defect identification or the examination of the effect of filtering and processing historical data to create lower inter-channel variance and allow earlier detection of defect data series. Some scope may also exist for machine learning opportunities: despite the limited size of the dataset available, it may be possible to incorporate wider reactor parameters and inter-channel correlations into more sophisticated models and this is an active area of research.

\section{Acknowledgements}

The contents, including any opinions and/or conclusions expressed, are those of the authors alone. We wish to thank Bruce Power for their financial and technical support of this research.

\section{References}

[1] Garland, W. J., Chaplin, R., Marleau, G., Nichita, E., Rouben, B., Popov, N. K., Hepburn, A., Jiang, J., Waller, E., Snell, V., Lister, D. H., Cook, W. G., Gacesa, M., Tayal, M., and Damario, D., 2014. The Essential $C A N D U$, first ed. University Network of Excellence in Nuclear Engineering, Hamilton, Ontario, Canada.

[2] IAEA, 2010. Review of Fuel Failures in Water Cooled Reactors, Nuclear Energy Series No. NF-T-2.1. Tech. rep., International Atomic Agency, Vienna.

[3] AECL CANDU, 1993. Design Manual: Failed Fuel Channel Identification System (Delayed Neutron Monitor System). Tech. rep., AECL CANDU, Mississauga, Ontario, Canada.

[4] Lewis, B. J., Chan, P. K., El-Jaby, A., Iglesias, F. C., and Fitchett, A., 2017. "Fission product release modelling for application of fuel-failure monitoring and detection - An overview". Journal of Nuclear Materials, 489, pp. 64-83.

[5] Lipsett, J. J., and Tseng, C. M., 1976. "Delayedneutron systems for failed fuel location in CANDU reactors". IEEE Transactions on Nuclear Science, 23(1), pp. 325-330.

[6] Schmeing, H., and Hogg, C., 2005. The Bruce B Delayed Neutron (DN) Monitoring System for Failed Fuel Detection: Twenty years of operating experience, recent improvement and planned upgrades. Tech. rep.

[7] MacDonald, R. D., Floyd, M. R., Lewis, B. J., Manzer, A. M., and Truant, P. T., 1990. Detecting, locating and identifying failed fuel in Canadian power reactors. 
Tech. rep., Chalk River Nuclear Laboratories, Chalk River, Ontario, Canada.

[8] Manzer, A. M., Sejnoha, R., Steed, R., Whynot, T., Graham, N., Barr, A., and Carter, T., 1992. "Fuel Defect Investigation at Point Lepreau". In Third International Conference on CANDU Fuel, Canadian Nuclear Society.

[9] Park, H., Manzer, A. M., Palleck, S., and Love, J., 1997. "Fuel defect root cause investigation at Wolsong1 ". In 5th International Conference on CANDU Fuel., pp. 21-25.

[10] Gheorghiu, E., and Gheorghiu, C., 2007. "Defected Fuel Monitoring at Cernavoda Nuclear Power Plant". In Nuclear Energy for New Europe, Nuclear Society of Slovenia.

[11] Fatima, T., Scientist, P., Qasim, K. G., and Engineer, P., 2002. "KANUPP Fuel Performance and Operational Experience". In International conference on WWER fuel performance, modelling and experimental support.

[12] Graham, R., and Stevens, J., 1974. Experience with CANDU reactors outside of Canada. Tech. rep.

[13] Livingstone, S. J., 2012. "Development of an on-line fuel failure monitoring system for CANDU reactors". PhD thesis, Royal Military College of Canada.

[14] Fitchett, A., 2012. "Assessing the impact of transport time between failed fuel and delayed neutron detectors in CANDU reactors". PhD thesis, Royal Military College of Canada.

[15] Page, E., 1954. "Continuous Inspection Schemes". Biometrika, 41(1), pp. 100-115.

[16] Huang, Y., Zhang, Y., Xu, B., Wu, Z., and Chambers, J. A., 2018. "A New Adaptive Extended Kalman Filter for Cooperative Localization". IEEE Transactions on Aerospace and Electronic Systems, 54(1), pp. 353-368.

[17] Gardner, E., 1985. "Exponential smoothing: The state of the art". International Journal of Forecasting, 4, pp. 1-28.

\section{Table caption list}

1. Summary of improvements enabled by exponential smoothing approach

\section{Figure Caption List}

1. A representative DN plot, with defect channel highlighted

2. General approach for the analysis of DN data

3. Calculated metrics for defect example 1

4. Calculated metrics for defect example 2

5. Cumulative deviations from mean 5, 10, 15 \& 20 scans from original defect identification point: defect example 1

6. $2 \mathrm{xN} \& \mathrm{ES}$ data for defect example 3, original history

7. $2 x N \&$ ES data for defect example 3 , extended history

8. $2 \mathrm{xN} \& \mathrm{ES}$ data for defect example 4, extended history

9. Mean, median \& standard deviation of raw counts for a single unit
10. Heatmap of normalized counts, scaled by monitor, shaded by standard deviation differences from mean 